\title{
A Giant Ruptured Noncoronary Sinus of Valsalva Aneurysm: A Case Report
}

\author{
Xueshan Zhao, MD, Lijie Jiang, MD, Honghua Yue, MD, Zhong Wu, MD \\ Department of Cardiovascular Surgery at West China Hospital, Sichuan University, Chengdu, Sichuan, People's Republic of China
}

\section{ABSTRACT}

The occurrence of a giant ruptured aneurysm originating from the noncoronary sinus of Valsalva in the right atrium is extremely rare. Herein, a case is presented of a giant ruptured noncoronary sinus of Valsalva aneurysm (SVA) that was protruding into the right atrium, which was almost completely occupied by an aneurysm. A 61-year-old female was referred to the hospital for exertional palpitation and dyspnea. While a surgical repair was performed by resection of the aneurysm and a sinus remodeling with a patch of fresh bovine pericardium, a very rare case was observed. It was a giant ruptured noncoronary sinus of aneurysm that completely occupied the right atrium, which was difficult to distinguish from the coronary aneurysm. It is also believed that various imaging examinations, such as cardiac computed tomography angiogram (CCTA) and transthoracic echocardiogram (TTE), were useful for the diagnosis.

\section{INTRODUCTION}

Giant ruptured sinus of Valsalva aneurysm is rarely observed. A 61-year-old female, who was associated with an exertional palpitation and dyspnea, was detected to have a giant ruptured noncoronary SVA by an echocardiography scan. The right atrium was almost completely occupied by the aneurysm, which was difficult to be distinguished from a coronary aneurysm. Eventually, the aneurysm was surgically repaired to reduce the right atrial pressure.

\section{CASE REPORT}

A 61-year-old female patient with a four-month history of dyspnea and exertional palpitation was admitted to the department. The patient occasionally experienced chest tightness, dizziness, and fatigue. No murmur was found in the

Received December 7, 2020; received in revised form fanuary 2, 2021; accepted fanuary 26, 2021.

Correspondence: Zhong Wu, Department of Cardiovascular Surgery, West China Hospital, Sichuan University, Chengdu, Sichuan, P.R. China, 610000, Telephone +86-028-85422897, Fax +86-028-85422897 (e-mail: wuzhong71@scu.edu.cn) cardiac auscultation area. The stage of heart failure was New York Heart Association (NYHA) class II. A chest roentgenography showed a convex contour of the right superior mediastinum. Electrocardiography revealed a sinus rhythm, and the results of the blood tests were normal. A computed tomography and angiography confirmed that there was a mass opacity (approximately $85 \mathrm{~mm} \times 76 \mathrm{~mm}$ ) in the right atrium (Figure 1A, 1B). (Figure 1) TTE revealed a large mass originating from the aortic noncoronary sinus that almost completely occupied the right atrium (Figure 1C). There was significant blood flow from the aorta to the aneurysm cavity, and a mild aortic valve regurgitation was observed. A routine median sternotomy was carried out. A cardiopulmonary bypass was performed via single aortic cannula, together with a 2-stage venous cannulation. After achieving cardiac arrest with intermittent retrograde and antegrade cold blood cardioplegic techniques, the ascending aorta was transected at the level of the sinotubular junction. Following a pericardiotomy, a giant aneurysmal dilation of the noncoronary sinus was exposed. It occupied almost the entire pericardial cavity, leading to a compression of the right ventricle and right atrium (Figure 1D). The ruptured aneurysm was excised, and the orifice was reconstructed with a patch of pericardium (Figure 1E). Transesophageal echocardiography (TEE) followed weaning from the cardiopulmonary bypass and showed no blood flow between the noncoronary sinus and the aneurysm body (Figure 1F). The patient demonstrated an uneventful course and was discharged on postoperative day 10 .

\section{DISCUSSION}

SVA is a congenital or acquired cardiac defect with an incidence rate of approximately $0.09 \%$ in the general population. It can be incidentally detected during cardiac imaging [Weinreich 2015]. Although there is ongoing debate concerning whether an acquired or congenital type is more common, the occurrence of SVA is due to the absence of normal elastic tissue within the aortic media. Based on this structural anomaly, a blind-ended diverticulum first is formed and then gradually increases in size until rupture. Thus, the anatomical structure of an SVA can directly affect its rupture points. SVA usually affects the right coronary sinus, followed by the noncoronary sinus, and finally the left coronary sinus [Feldman 2006]. An SVA often ruptures into the adjacent cardiac chambers, while noncoronary sinus often ruptures into the 

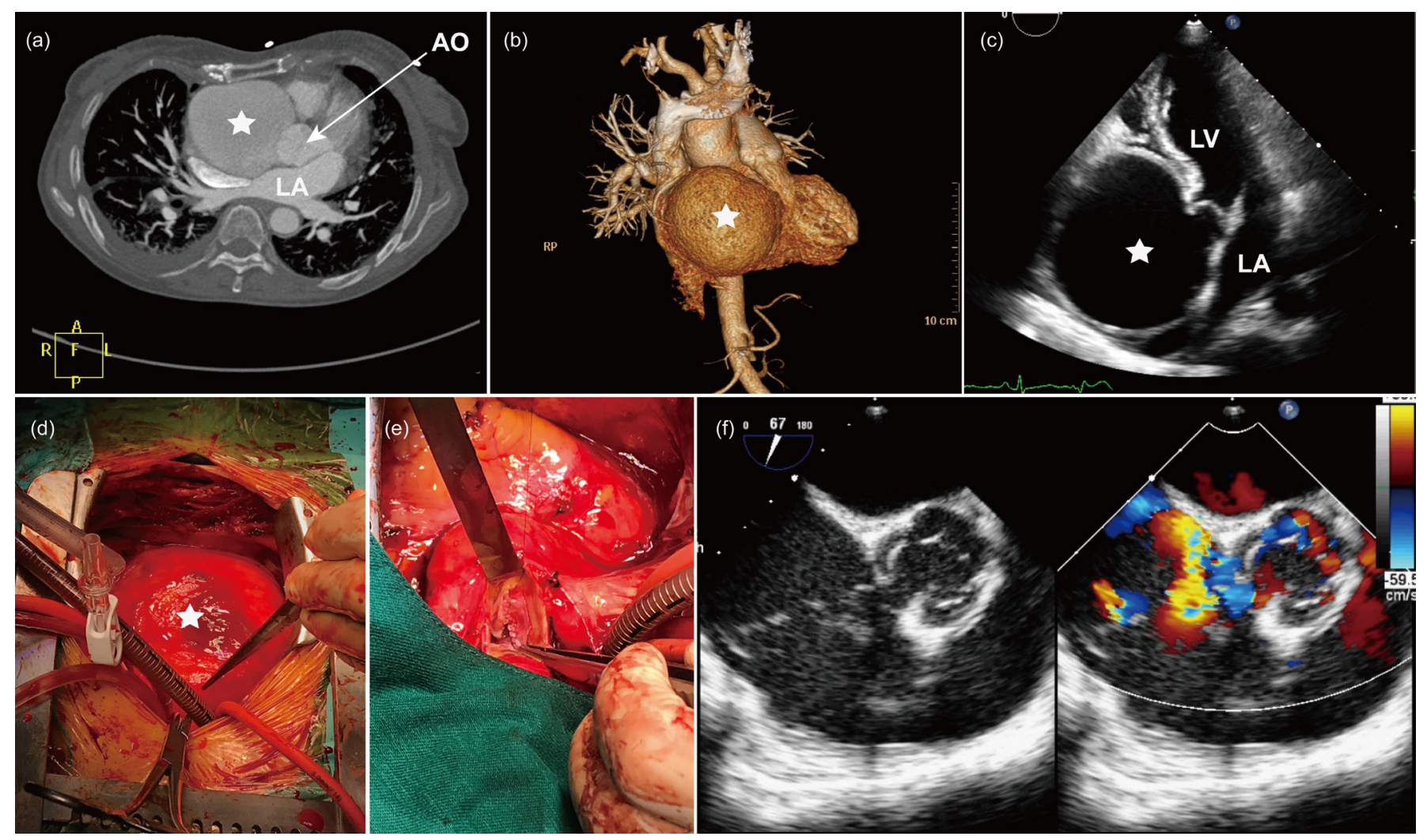

Figure 1. A, B) Computed tomography angiography revealed a giant aneurysm of the noncoronary sinus of Valsalva with maximum dimensions of 85 mm $\times 76$ $\mathrm{mm}$. C) TTE revealed the large mass originated from the aortic noncoronary sinus and almost completely occupied the right atrium. D) Intraoperative images. The surgery confirmed the presence of a giant noncoronary sinus of Valsalva aneurysm. E) The ruptured aneurysm was excised, and the orifice was reconstructed with a patch of pericardium. F) TEE followed by weaning from cardiopulmonary bypass showed no blood flow between the noncoronary sinus and the aneurysm body. AO: aorta; LA: left atrium; LV: left ventricle; asterisk: aneurysm

right atrium. Reports about the occurance of a giant ruptured noncoronary sinus of Valsalva aneurysm are extremely rare. Rupture of the right noncoronary sinus often leads to a shunt between the right atrium and aorta. The pathological features of a congenital SVA most likely are related to connective tissue disorders, while many infectious etiologies, such as bacterial endocarditis, syphilis and tuberculosis, also tend to lead to its formation.

SVA cases usually are asymptomatic, and most incidentally are detected before and after a rupture. In addition to its location and size, the speed of a rupture is the main determinant of its clinical outcome [Weinreich 2015]. In the case of an aneurysm rupture, common symptoms may include abdominal pain, substernal pain, and varying degrees of dyspnea. In many cases, an acute cardiac failure, hemodynamic dysfunction cardiac tamponade, and sudden cardiac arrest could have been reported [Weinreich 2015; Yan 2014]. The patient was found to have a ruptured SVA during examination for the onset of an exertional dyspnea.

Numerous imaging methods can be used to diagnose patients with SVA. Echocardiography is useful for assessing the size, shape, and location of an aortic sinus aneurysm as well as the rupture of cardiac cavity and whether there are other cardiac malformations. It should be noticed that the sensitivity of this method is greater than $98 \%$. Thus, echocardiography has become the first choice for diagnosing SVA and distinguishing it from other heart defects. While an echocardiogram is the conventional imaging technique for this kind of finding, CCTA has progressively been utilized. In addition, aortic angiography, which has been considered the gold standard for assessing an aortic sinus aneurysm rupture, can be used to identify the morphology, size, and location of the rupture.

As soon as a ruptured SVA is identified, surgery should be done as quickly as possible. Heart failure should not be an absolute contraindication to surgery [Au 1998; Hamid 1994]. After the removal of SVA, a primary closure and patch closure are considered to be two main approaches for wound closure. A primary closure has the risk of a residual shunt, while repairing the sinus wall with a patch can reduce tension and prevent recurrence. Hence, the sinus orifice of the patient was reconstructed with a pericardial patch.

\section{CONCLUSION}

We experienced a very rare case of a ruptured giant noncoronary sinus of aneurysm that completely occupied the right atrium and was difficult to distinguish from a coronary 
aneurysm. Various imaging examinations such as CCTA and TTE were useful for the diagnosis.

\section{REFERENCES}

Au WK, Chiu SW, Mok CK, Lee WT, Cheung D, He GW. 1998. Repair of ruptured sinus of Valsalva aneurysm: determinants of long-term survival. The Annals of thoracic surgery. 66(5):1604-1610.

Feldman DN, Roman MJ. 2006. Aneurysms of the sinuses of Valsalva.
Cardiology. 106(2):73-81.

Hamid IA, Jothi M, Rajan S, Monro JL, Cherian KM. 1994. Transaortic repair of ruptured aneurysm of sinus of Valsalva. Fifteen-year experience. The Journal of thoracic and cardiovascular surgery. 107(6):1464-1468.

Weinreich M, Yu PJ, Trost B. 2015. Sinus of Valsalva aneurysms: review of the literature and an update on management. Clinical cardiology. 38(3):185-189.

Yan F, Abudureheman M, Huo Q, Shabiti A, Zhu T, Liu Z. 2014. Surgery for sinus of Valsalva aneurysm: 33 -year of a single center experience. Chinese medical journal. 127(23):4066-4070. 